Appl. Set-Valued Anal. Optim. 2 (2020), No. 2, pp. 175-181

Available online at http://asvao.biemdas.com

https://doi.org/10.23952/asvao.2.2020.2.04

\title{
FIXED POINT RESULTS FOR MULTI-VALUED LOCALLY CONTRACTIVE OPERATORS
}

\author{
ADRIAN PETRUŞEL ${ }^{1,2, *}$, GABRIELA PETRUŞEL $^{3}$ \\ ${ }^{1}$ Department of Mathematics, Babes-Bolyai University Cluj-Napoca, Romania \\ ${ }^{2}$ Academy of Romanian Scientists, Bucharest, Romania \\ ${ }^{3}$ Department of Business, Babeş-Bolyai University Cluj-Napoca, Romania
}

\begin{abstract}
In this paper, we present several fixed point results for multi-valued $(\varepsilon, \alpha)$-uniformly locally contractive operators in complete $\varepsilon$-chainable metric spaces. Connections to the mathematics of multi self-similar sets are given.

Keywords. Multi-valued operator; $\varepsilon$-chainable metric space; Fixed point; Multi-valued contraction; Multi-valued locally contractive operator.
\end{abstract}

\section{INTRODUCTION}

Let $(X, d)$ be a metric space. We denote by $P(X)$ the family of all nonempty subsets of $X$, by $P_{c l}(X)$ the family of all nonempty closed subsets of $X$, by $P_{b}(X)$ the family of all nonempty bounded subsets of $X$, and by $P_{c p}(X)$ the family of all nonempty compact subsets of $X$.

The following notations will be used throughout this paper:

(1) the gap functional generated by $d$,

$$
D_{d}(A, B):=\inf \{d(a, b) \mid a \in A, b \in B\} ;
$$

(2) the excess functional of $A$ over $B$ generated by $d$,

$$
e_{d}(A, B):=\sup \left\{D_{d}(a, B) \mid a \in A\right\} ;
$$

(3) the Hausdorff-Pompeiu functional generated by $d$,

$$
H_{d}(A, B)=\max \left\{e_{d}(A, B), e_{d}(B, A)\right\} .
$$

The diameter of a set $Y \in P(X)$ will be denoted by

$$
\operatorname{diam}_{d}(Y):=\sup _{x, y \in Y} d(x, y) .
$$

We will avoid the subscript $d$ if no confusion occurs.

A metric space $(X, d)$ is called $\varepsilon$-chainable if $\varepsilon>0$ and, for every $x, \tilde{x} \in X$, there exists an $\varepsilon$ chain, i.e., a finite set of points $x=x_{0}, x_{1}, \ldots, x_{n}=\tilde{x}$ (where the natural number $n \geq 1$ may depend on both $u$ and $v$ ) from $X$ such that $d\left(x_{i-1}, x_{i}\right)<\varepsilon$, for each $i \in\{1,2, \ldots, n\}$. A well-chainable

${ }^{*}$ Corresponding author.

E-mail addresses: petrusel@math.ubbcluj.ro (A. Petruşel), gabi.petrusel@tbs.ubbcluj.ro (G. Petruşel).

Received April 20, 2020; Accepted May 31, 2020. 
metric space is an $\varepsilon$-chainable metric space with respect to any $\varepsilon>0$. For other details, we refer to [10].

In 1969, Nadler proved a fixed point result in complete $\varepsilon$-chainable metric spaces for multivalued locally contractive operators and extended some previous theorems given by Edelstein in [4] for the single-valued case. See also [7] for local fixed point theorems of single-valued operators.

A multi-valued operator $F: X \rightarrow P(X)$ is said to be a multi-valued $(\varepsilon, \alpha)$ - uniformly locally contractive operator if, $\varepsilon>0, \alpha \in[0,1[$ and the following implication holds

$$
x, y \in X \text { and } d(x, y)<\varepsilon \Rightarrow H(F(x), F(y)) \leq \alpha d(x, y) .
$$

A fixed point of operator $F$ is an element $x^{*} \in X$ such that $x^{*} \in F\left(x^{*}\right)$. A strict fixed point of $F$ is an element $x^{*} \in X$ such that $F\left(x^{*}\right)=\left\{x^{*}\right\}$. From now on, we will denote by $\operatorname{Fix}(F)$ the fixed point set of $F$ and by $\operatorname{SFix}(F)$ the set of all strict fixed points of $F$.

Theorem 1.1. [6] Let $\varepsilon>0$ and $(X, d)$ be a complete metric $\varepsilon$-chainable space. Suppose that $F: X \rightarrow P_{c p}(X)$ is a multi-valued $(\varepsilon, \alpha)$ - uniformly locally contractive operator. Then $\operatorname{Fix}(F) \neq \emptyset$.

For the case of well-chained metric space, we refer to [5]. For the case of the sequence of multi-valued operators and fixed points, we refer to [2]. For other interesting fixed point theorems for multi-valued locally contractive type operators, we refer to [11] and [12]. For other fixed point results for multi-valued operators, see [1] and [8].

In 1983, Barcz extended the above result for the case of nonlinear contractions with bounded and closed values. Denote by $\Psi$ the family of all functions $\psi: \mathbb{R}_{+} \rightarrow \mathbb{R}_{+}$, which are increasing, continuous from the right and satisfy the following two conditions:

(i) $\sum_{n \geq 0} \psi^{n}(t)<\infty$;

(ii) $\psi(t)=0$ implies $t=0$.

A multi-valued operator $F: X \rightarrow P(X)$ is said to be a multi-valued nonlinear $(\varepsilon, \psi)$-uniformly locally contractive operator if, $\varepsilon>0, \psi \in \Psi$ and the following implication holds

$$
x, y \in X \text { and } d(x, y)<\varepsilon \Rightarrow H(F(x), F(y)) \leq \psi(d(x, y)) .
$$

Theorem 1.2. [3] Let $\varepsilon>0$ and $(X, d)$ be a complete metric $\varepsilon$-chainable space. Suppose that $F: X \rightarrow P_{b, c l}(X)$ is a multi-valued $(\varepsilon, \psi)$-uniformly locally contractive operator. Then $\operatorname{Fix}(F) \neq \emptyset$.

The purpose of this paper is to give several fixed point results for multi-valued $(\varepsilon, \alpha)$-uniformly locally contractive operators in complete $\varepsilon$-chainable metric spaces. Connections to the mathematics of multi self-similar sets are also given.

\section{MAIN RESUlTS}

Let $(X, d)$ be a metric space and $F: X \rightarrow P(X)$ be a multi-valued operator. We say that $\left(x_{n}\right)_{n \in \mathbb{N}} \subset X$ is a sequence of successive approximations for $F$ starting from $x_{0}$ if $x_{n+1} \in F\left(x_{n}\right)$, for every $n \in \mathbb{N}$. For related notions, we refer to [6], [9] and [12].

The following result is a fixed point theorem for an $(\varepsilon, \alpha)$-uniformly locally contractive operator in a complete metric space $(X, d)$. It follows immediately from [3, Theorem 3.1]. We notice that the boundedness of the values of the multi-valued operator can be omitted. 
Theorem 2.1. Let $(X, d)$ be a complete metric space and let $F: X \rightarrow P_{c l}(X)$ be a multivalued $(\varepsilon, \alpha)$-uniformly locally contractive operator. Suppose there exists $x_{0} \in X$ such that $D\left(x_{0}, F\left(x_{0}\right)\right)<\varepsilon$. Then, the following conclusions hold:

(a) Fix $(F) \neq \emptyset$;

(b) there exists a sequence $\left(x_{n}\right)_{n \in \mathbb{N}}$ of successive approximations for $F$ starting from $x_{0} \in X$, which converges to a fixed point $x^{*}\left(x_{0}\right)$ of $F$.

(c) the following apriori estimation holds

$$
d\left(x_{n+1}, x^{*}\left(x_{0}\right)\right) \leq \frac{(q \alpha)^{n}}{1-q \alpha} d\left(x_{0}, x_{1}\right), \text { for every } n \in \mathbb{N},
$$

where $q \in] 1, \frac{1}{\alpha}[$ can be arbitrary chosen.

Proof. Lettting $\psi(t)=\alpha t$ for every $t \in \mathbb{R}_{+}$in [3, Theorem 3.1], we obtain (a) and (b). For (c), by a classical approach, we have

$$
d\left(x_{n}, x_{n+p}\right) \leq \frac{(q \alpha)^{n}}{1-q \alpha} d\left(x_{0}, x_{1}\right), \text { for every } n \in \mathbb{N} \text { and } p \in \mathbb{N}^{*}
$$

Letting $p \rightarrow \infty$, we get the desired conclusion immediately.

Using the properties of the excess functional $e$, we can prove the following slight extension of the above theorem in a similar approach.

Theorem 2.2. Let $(X, d)$ be a complete metric space and $F: X \rightarrow P_{c l}(X)$ be a multi-valued operator for which there exist $\varepsilon>0$ and $\alpha \in[0,1[$ such that the following implication holds

$$
x, y \in X \text { with } d(x, y)<\varepsilon \Rightarrow e(F(x), F(y)) \leq \alpha d(x, y) .
$$

Then, the following conclusions hold:

(a) Fix $(F) \neq \emptyset$;

(b) there exists a sequence $\left(x_{n}\right)_{n \in \mathbb{N}}$ of successive approximations for $F$ starting from $x_{0}$, which converges to a fixed point of $F$ and we have the following apriori estimation

$$
d\left(x_{n+1}, x^{*}\left(x_{0}\right)\right) \leq \frac{(q \alpha)^{n}}{1-q \alpha} d\left(x_{0}, x_{1}\right), \text { for every } n \in \mathbb{N},
$$

where $q \in] 1, \frac{1}{\alpha}[$ can be arbitrary chosen.

If $(X, d)$ is a metric space and $F: X \rightarrow P_{c p}(X)$ is a multi-valued upper semi-continuous operator, then the fractal operator generated by $F$ is denoted by $\hat{T}: P_{c p}(X) \rightarrow P_{c p}(X)$ and defined by

$$
\hat{T}(Y):=F(Y)=\bigcup_{y \in Y} F(y), \text { for all } Y \in P_{c p}(X) .
$$

A fixed point of $\hat{T}$ is called a multi self-similar set for $T$. In the same context, if $F_{i}: X \rightarrow P_{c p}(X)$ (where $i \in\{1,2, \cdots, m\}$ ) are multi-valued upper semi-continuous operators, then we denote by

$$
\hat{T}_{F}: P_{c p}(X) \rightarrow P_{c p}(X)
$$

given by

$$
\hat{T}_{F}(Y):=\bigcup_{i=1}^{m} F_{i}(Y), \text { for } Y \in P_{c p}(X)
$$


the fractal operator generated by the iterated multi-function system

$$
F=\left(F_{1}, \cdots, F_{m}\right) \text {. }
$$

By the upper semi-continuity of each $F_{i}(i \in\{1,2, \cdots, m\})$, the operator $\hat{T}_{F}$ is well-defined. It is easy to observe that any multi-valued $(\varepsilon, \alpha)$-uniformly locally contractive operator $F: X \rightarrow$ $P_{c p}(X)$ is upper semi-continuous.

Recall that a single-valued operator $f: X \rightarrow X$ is said to be an $(\varepsilon, \alpha)$-uniformly locally contraction if, $\varepsilon>0, \alpha \in[0,1[$ and the following implication holds

$$
x, y \in X, x, y<\varepsilon \Rightarrow d(f(x), f(y)) \leq \alpha d(x, y) .
$$

In the single-valued case, the following result was proved by Nadler [6]

Theorem 2.3. [6] Let $(X, d)$ be a complete $\varepsilon$-chainable metric space and let $f: X \rightarrow X$ be an $(\varepsilon, \alpha)$-uniformly locally contraction. Then, there exists a unique fixed point $x^{*} \in X$ of $f$ and, for each $x \in X$, the sequence of Picard iterations $\left(f^{n}(x)\right)_{n \in \mathbb{N}}$ converges to $x^{*}$ as $n \rightarrow \infty$.

In the multi-valued case, we have the following result.

Theorem 2.4. Let $(X, d)$ be a metric space and let $F: X \rightarrow P_{c p}(X)$ be an $(\varepsilon, \alpha)$-uniformly locally contractive operator. Then, the fractal operator $\left.\hat{T}: P_{c p}(X) \rightarrow P_{c p}(X)\right)$ generated by $F$ is an $(\varepsilon, \alpha)$-uniformly locally contraction on $P_{c p}(X)$.

Proof. We show that if $A, B \in P_{c p}(X)$ with $H(A, B)<\varepsilon$, then

$$
H(\hat{T}(A), \hat{T}(B)) \leq \alpha H(A, B) .
$$

For this purpose, let $u \in \hat{T}(A)$ be arbitrary. Then, there exists $a \in A$ such that $u \in F(a)$. For $a \in A$, by the compactness of $B$, there exists $b \in B$ such that

$$
d(a, b) \leq H(A, B)<\varepsilon .
$$

By the compactness of the values of $F$, let $v \in F(b) \subset \hat{T}(B)$ such that

$$
d(u, v)=D(u, F(b)) .
$$

Thus, using the fact that $F: X \rightarrow P_{c p}(X)$ is $(\varepsilon, \alpha)$-uniformly locally contractive, we conclude

$$
\begin{aligned}
d(u, v) & =D(u, F(b)) \\
& \leq H(F(a), F(b)) \\
& \leq \alpha d(a, b) \\
& \leq \alpha H(A, B) .
\end{aligned}
$$

By a similar method, we can also show that, for every $v \in \hat{T}(B)$, there exists $u \in \hat{T}(A)$ such that $d(u, v) \leq \alpha H(A, B)$. Hence

$$
H(\hat{T}(A), \hat{T}(B)) \leq \alpha H(A, B)
$$

Before our next result, we recall the following important theorem proved by $\mathrm{Xu}$ in [12].

Theorem 2.5. If $(X, d)$ is an $\varepsilon$-chainable metric space, then $\left(P_{c p}(X), H_{d}\right)$ is also an $\varepsilon$-chainable metric space. 
Using Theorem 2.3 and the above two Theorems, we immediately obtain the following existence, uniqueness and approximation result.

Theorem 2.6. Let $(X, d)$ be a complete $\varepsilon$-chainable metric space and let $F: X \rightarrow P_{c p}(X)$ be an $(\varepsilon, \alpha)$-uniformly locally contractive operator. Then, there exists a unique $A^{*} \in P_{c p}(X)$ such that $A^{*}=F\left(A^{*}\right)$ (i.e., a multi self-similar set) and the sequence $\left(A_{n}\right)_{n \in \mathbb{N}} \subset P_{c p}(X)$, defined by $A_{n+1}:=F\left(A_{n}\right), n \in \mathbb{N}$ converges to $A^{*}$ as $n \rightarrow \infty$, for every $A_{0} \in P_{c p}(X)$.

Proof. By Theorem 2.4, the operator $\left.\hat{T}: P_{c p}(X) \rightarrow P_{c p}(X)\right)$ is an $(\varepsilon, \alpha)$-uniformly locally contraction. Using Theorem 2.3 and Theorem 2.5, we get that $\hat{T}$ has a unique fixed point $A^{*} \in P_{c p}(X)$ and the corresponding sequence of successive approximations starting from any $A_{0} \in P_{c p}(X)$ converges to $A^{*}$. This completes the proof.

Example 2.1. Let $X:=\left\{(x, y) \in \mathbb{R}^{2}: x=\cos t, y=\sin t, t \in\left[0, \frac{3 \pi}{2}\right]\right\}$ and $F: X \rightarrow P_{c p}(X)$ be a mapping defined by

$$
F(\cos t, \sin t)=\left\{(1,0),\left(\cos \frac{t}{2}, \sin \frac{t}{2}\right)\right\} .
$$

Then, $F$ is a multi-valued $(\varepsilon, \lambda)$-uniformly locally contractive operator and it is not a global contraction. Since $X$ is complete $\varepsilon$-chainable, Theorem 2.6 applies and $F$ has a unique fixed point $A^{*}=\{(1,0)\} \in X \times X$ and the sequence of Picard iteration given by

$$
F^{n}(\cos t, \sin t)=\left\{(1,0),\left(\cos \frac{t}{2^{n}}, \sin \frac{t}{2^{n}}\right)\right\}
$$

converges to $\{(1,0)\}$ as $n \rightarrow \infty$, for every $t \in\left[0, \frac{3 \pi}{2}\right]$.

Let us consider now an iterated multi-function system $F=\left(F_{1}, \cdots, F_{m}\right)$, where $F_{i}: X \rightarrow$ $P_{c p}(X)$ (for $i \in\{1,2, \cdots, m\}$ ) are multi-valued $(\varepsilon, \alpha)$-uniformly locally contractive operators.

Theorem 2.7. Let $(X, d)$ be a metric space. Suppose that $F_{i}: X \rightarrow P_{c p}(X)$ are $\left(\varepsilon, \alpha_{i}\right)$-uniformly locally contractive operators, for $i \in\{1,2, \cdots, m\}$. Then, the fractal operator $\hat{T}_{F}: P_{c p}(X) \rightarrow$ $P_{c p}(X)$, generated by the iterated multi-function system $F=\left(F_{1}, \cdots, F_{m}\right)$ is an $(\varepsilon, \alpha)$-uniformly locally contraction, where $\alpha:=\max \left\{\alpha_{1}, \cdots, \alpha_{m}\right\}$.

Proof. First, notice that, by the upper semi-continuity of each $F_{i}$, the operator $\hat{T}_{F}$ is well defined. We have to show that if $A, B \in P_{c p}(X)$ with $H(A, B)<\varepsilon$, then

$$
H\left(\hat{T}_{F}(A), \hat{T}_{F}(B)\right) \leq \lambda H(A, B) .
$$

For this purpose, let $u \in \hat{T}_{F}(A)$. Then, there exists $k \in\{1,2, \cdots, m\}$ and $a \in A$ such that $u \in$ $F_{k}(a)$. For $a \in A$, by the compactness of the set $B$, there exists $b \in B$ such that

$$
d(a, b) \leq H(A, B)<\varepsilon .
$$

Let $v \in F_{k}(b) \in \hat{T}_{F}(B)$ be such that

$$
d(u, v)=D\left(u, F_{k}(b)\right) .
$$

Then, using the fact that $F_{k}: X \rightarrow X$ is $\left(\varepsilon, \lambda_{k}\right)$-uniformly locally contractive, we get

$$
\begin{aligned}
d(u, v) & =D\left(u, F_{k}(b)\right. \\
& \leq H\left(F_{k}(a), F_{k}(b)\right) \\
& \leq \alpha_{k} d(a, b) \\
& \leq \alpha H(A, B) .
\end{aligned}
$$


Using a similar method, we can also show that, for every $v \in \hat{T}_{F}(B)$, there exists $u \in \hat{T}_{F}(A)$ such that $d(u, v) \leq \alpha H(A, B)$. Hence

$$
H\left(\hat{T}_{F}(A), \hat{T}_{F}(B)\right) \leq \alpha H(A, B) .
$$

This completes the proof.

Using the above results, we get the following existence, uniqueness and approximation result.

Theorem 2.8. Let $(X, d)$ be a complete $\varepsilon$-chainable metric space and let $F_{i}: X \rightarrow P_{c p}(X)$ be $\left(\varepsilon, \alpha_{i}\right)$-uniformly locally contractive operators, for $i \in\{1,2, \cdots, m\}$. Then, there exists a unique $A^{*} \in P_{c p}(X)$ such that $A^{*}=\bigcup_{i=1}^{m} F_{i}\left(A^{*}\right)$ and the sequence $\left(A_{n}\right)_{n \in \mathbb{N}} \subset P_{c p}(X)$, defined by

$$
A_{n+1}:=\bigcup_{i=1}^{m} F_{i}\left(A_{n}\right),
$$

$n \in \mathbb{N}$ converges to $A^{*}$ as $n \rightarrow \infty$, for every $A_{0} \in P_{c p}(X)$.

Proof. From Theorem 2.7, we have that the operator $\left.\hat{T}_{F}: P_{c p}(X) \rightarrow P_{c p}(X)\right)$, given by

$$
\hat{T}_{F}(Y):=\bigcup_{i=1}^{m} F_{i}(Y),
$$

is an $(\varepsilon, \alpha)$-uniformly locally contraction. Using Theorem 2.3 and Theorem 2.5, we get that the operator $\hat{T}_{F}$ has a unique fixed point $A^{*}$ in $P_{c p}(X)$ and the corresponding sequence of successive approximations starting from any $A_{0} \in P_{c p}(X)$ converges to $A^{*}$. This completes the proof.

Remark 2.1. It is an open question to prove the similar results for the case of multi-valued nonlinear $(\varepsilon, \psi)$-uniformly locally contractive operators or to other classes of contractive type multi-valued operators. Another question is to extend the above results for multi-valued operators with closed values.

\section{Acknowledgments}

The authors were supported by the Babeş-Bolyai University Cluj-Napoca under ATC grants.

\section{REFERENCES}

[1] C.D. Alecsa, A. Petruşel, On some fixed point theorems for multi-valued operators by altering distance technique, J. Nonlinear Var. Anal. 1 (2017), 237-251.

[2] A. Azam, M. Arshad, Fixed points of a sequence of locally contractive multivalued maps, Computers Math. Appl. 57 (2009), 96-100.

[3] E. Barcz, Fixed point theorems for multi-valued mappings, Demonstratio Math. 16 (1983), 735-744.

[4] M. Edelstein, An extension of Banach's contraction principle, Proc. Amer. Math. Soc. 12 (1961), 7-10.

[5] P.K.F. Kuhfitting, Fixed point of locally contractive and non-expansive set-valued mappings, Pacific J. Math. 65 (1976), 399-403.

[6] S.B. Nadler Jr., Multi-valued contraction mappings, Pacific J. Math. 30 (1969), 475-488.

[7] A. Petruşel, Local fixed point results for graphic contractions, J. Nonlinear Var. Anal. 3 (2019), 141-148.

[8] A. Petruşel, G. Petruşel, On Reich's strict fixed point theorem for multi-valued operators in complete metric spaces, J. Nonlinear Var. Anal. 2 (2018), 103-112.

[9] I.A. Rus, A. Petruşel, G. Petruşel, Fixed Point Theory, Cluj University Press, Cluj, 2008. 
[10] K. Shrivastava, G. Agarwal, Characterization of $\varepsilon$-chainable sets in metric spaces, J. Indian Pure Appl. Math. 33 (2002) 933-940.

[11] H.K. Xu, $\varepsilon$-chainability and fixed points of set-valued mappings in metric spaces, Math. Japonica 39 (1994), 353-356.

[12] H.K. Xu, Metric fixed point theory for multivalued mappings, Diss. Math. 389 (2000), 1-39. 